\title{
A Corpus-based Study of Valency Sentence Patterns of English Verbs*
}

\author{
Guobing Liu \\ Faculty of International Studies, Henan Normal University, Xinxiang, China \\ Yaping Du \\ Faculty of International Studies, Henan Normal University, Xinxiang, China
}

\begin{abstract}
Based on the COCA, this paper investigates valency sentence patterns of the English verb APPOINT from the perspective of syntactic valency. And it analyzes the dominated components of verbs with the corpus linguistic method of collocation. It has been found: (1) The verb APPOINT has seven valency sentence patterns identified in the active sentences and fifteen patterns in the passive sentences. (2) The complement types associated with the verb APPOINT include subject complement, object complement, nominal complement with or without as, verbal complement with an infinitive or with to-be followed by a noun or a noun phrase, prepositional complement with the preposition to, by or for. (3) There is regularity existing in the complements. This present study describes valency sentence patterns of verbs, taking the sentence as the smallest research unit and verbs as the core of the sentence. The research results provide a new sight for second language teaching, especially for English vocabulary teaching.
\end{abstract}

Index Terms - valency theory, valency sentence pattern, corpus

\section{INTRODUCTION}

Local grammar has become a new and hot research field for corpus linguistics. It is a descriptive grammatically path for specific language use, where it uses corpus and computational linguistics methods to describe typically communicative functions or special expressions in various genres. Its purpose is to meet the need for automatic information processing of a large number of authentic texts. The emergence of local grammars on the one hand could compensate for the disadvantages of general grammar in describing the actual language. On the other hand, it provides a new research perspective of the phraseology, natural language processing and even discourse analysis (Zhang \& Wei, 2017). And valency represents a local grammar which is concerned with the lexicalization of syntax, often called lexico-grammar (Richard, 2014). The concept of valency originates from the valency theory; it reflects the dominated ability of verbs, adjectives, and nouns with valency requirements on other lexical items. By describing the valency of these words, their syntactic and semantic competencies could be made a succinct description. Therefore, valency theory has become an effective research approach to the language structure (Yuan, 2010).

The previous research on valency theory is largely conducted on the basis of introspective data from the perspective of top-down and the result solely presents the number of valency of words. This causes that it is not clear about how to use the words. Reichardt (2014) adopted the methodology of fusing corpus linguistics and valency theory and he explored valency sentence patterns of verbs and interrelationship of local grammar, meaning, and translation equivalence, taking the English verb CONSIDER as an example. The reason why corpus linguistics is fused is that corpus provides authentic texts and is found to be a tool in verifying sentence pattern. The features of valency pattern and valency relationship can be determined by observing the context of different language units in large corpora (Chen, 2009), because valency sentence pattern has something in common with pattern grammar (Sinclair, 1991; Hunston \& Francis, 2000) of corpus linguistics.

However, there is little research including the grammatical category of voice when studying verb valency structure. And language should be described truly and comprehensively. Therefore, this paper will investigate valency sentence patterns of verbs from the perspective of syntactic valency including the category of voice, taking the verb English APPOINT as an example. And it will analyze dominated components of verbs employed a corpus linguistic method of collocation. The results can facilitate the teaching of college English vocabulary and promote the authenticity and fluency of learners' second language.

\section{LITERATURE REVIEW}

\section{A. The Principle of Valency Theory}

Valency theory is a grammatical system which studies the valency of words. It is connected with the property of

\footnotetext{
${ }^{*}$ This research was supported in part by the Project of National Social Science Fund in China (Project No.: 14BYY084).
} 
words to combine with other elements in forming phrases and sentences. It is based on dependency relations, where the concern of linguistic investigation is the sentence. And valency properties of verbs are closely related to the overall structures of a clause or sentence (Herbst, 2009), in other words, the sentence complements are dependents of the main verb of a sentence or clause. Therefore, clause forms the smallest communicative entity. And the sentence is the smallest research unit in the studies of valency theory. What's more, the verb is taken as the core in a sentence and associates with other components to constitute one sentence. This association obeys the principle of hierarchy of verb being the dominant position and the other components subordinate in the sentence (Zhi \& Liu, 2000). Verbs have the power to dominate other word classes, which form the action elements. The number of valency of a verb depends on the number of its actions (Lu, 1996). Depending on how many actions a verb can occur with, generally its valency can be classified as zero-valent, monovalent, divalent, trivalent and, though very rare, tetravalent. The zero-valent verb means that it is not mandatory to associate with the action, and can express the complete meaning itself. The monovalent verbs can only dominate an action, which means that the verb cannot be followed by an object. The bivalent verb can only dominate two actions, that is, it can only associate an object. The trivalent verb can only dominate three actions, which means two objects follow the verb. Thus, the basic point of valency theory is the core of verbs. In addition, valency is not currently restricted to study verbs, but has been applied to subclasses of all word-classes, e.g. nouns or adjectives.

\section{B. Categorization by Syntactic Function}

A categorization of valency complements by syntactic function for sentence analysis concerns the distinction between subjects and objects in traditional grammar (Richard, 2004). In valency theory the term "complement" is used. It refers to any elements that are required by the regent to form a grammatically correct phrase or clause. Example a shows the expression of components.

a. $\quad \frac{\text { The president }}{\text { subject }}$ appoints the Council of Minster.
Traditional G. object
Valency G. subject complement

However, in order to describe valency sentence patterns of the verb succinctly, the term "complement" after the subject and the object is usually omitted. Example b shows the expression employed in this paper. More detailed description will be elaborated in the next section.
b. $\underline{\text { She }}$
appoints
subject
$\underline{\text { Gabriel }}$
$\underline{\text { the farm manager. }}$
object nominal complement

\section{Valency Sentence Pattern}

Depending on how many complement types a verb can occur with, its valency can be described as zero-valent , monovalent, divalent, trivalent and, though very rare, tetravalent, as shown below.

Monovalent: c. The teacher smiled.

Divalent: $\quad \mathrm{d}$. The workers are building the house.

Trivalent: e. My father bought me a book.

$$
<\mathrm{sub}>
$$

$<$ sub obj>

$<$ sub ind obj>

f. The manager appointed Mary to handle the

<sub obj verbal complement> crisis.

g. She appointed Gabriel her farm manager.

<sub obj nominal complement>

Tetravalent: h. We bought many apples from an old man $<<$ sub obj prp1 prp2> with 2 dollars.

From the examples presented above, it is found that there are several sentence realization patterns. The terms divalent, trivalent and tetravalent solely indicate that the verb requires two, three or four complements respectively, but they do not state the required type of complements. Therefore, a trivalent sentence pattern may have different realization, which can be with a subject, an object and indirect complement (e), or with a subject, an object and verbal complement (f), or with a subject, an object and a nominal complement (f).

Nevertheless, the valency complement types presented above are not detailed and succinct enough. Therefore sub-classification based on realization forms, such as finite or non-finite clauses, is introduced in the case study. Table I shows examples description of English sentences.

TABLE I.

DESCRIPTION OF VALENCY COMPLEMENT TYPES

\begin{tabular}{|c|c|c|c|}
\hline \multicolumn{1}{|c|}{ DESCRIPTION OF VALENCY COMPLEMENT TYPES } \\
\hline i. & We & like & making cakes. \\
\hline Complement type & Sub & V & Objing \\
\hline j. & Trustees & have been appointed & to administer the pension fund. \\
\hline Complement type & Sub & be V-ed & vb-to-inf \\
\hline
\end{tabular}

As can be seen the sentence (i) structure is <Sub V Objing>. This indicates the verb like is a divalent, which governs a subject complement and an object complement. Objing means that the object complement is realized with a ing-clause. The valency sentence (j) patterns is <Sub be V-ed vb-to-inf $\rangle$. It means that the verb appoint has two valencies. One is subject complement. The other is verbal complement, whose realisation is a to-inf-clause. What can 
also be known from the description of be V-ed is that the verb is used in a passive sentence. This paper will present valency sentence pattern of the verb APPOINT according to the descriptive system..

\section{Previous Studies on Valency Sentence Pattern}

Allerton (1982) presented 33 English valency sentence patterns and Fisher (1997) noted 59 German and 39 English valency sentence patterns. Richard (2014) explored the interrelationship of local grammar, meaning, and translation equivalence, using a case study of the English verb CONSIDER, and compared in a monolingual study with its near-synonyms BELIEVE, FEEL and THINK, and in a contrastive analysis with their German translation equivalents.

In recent years, some China scholars (Zhen \& Yang, 2015, 2016; Liu \& Du, 2017; Zhang, 2018) have applied valency theory to describe sentence valency patterns of English verbs, which makes the language description more concise and more conducive to learners' learning. Zhen and Yang (2015) attempted to develop a set of corpus-driven descriptive system of valency pattern of verbs, in which it not only retained the specific words and part of speech of pattern grammar, but also included the syntactic function of valency grammar. And analytical methods of corpus linguistics research were employed, for instance, collocation, semantic preference and semantic sequence. Taking the verb CONSIDER as an example, they used this descriptive system to analyze characteristics of verb valency in Chinese Learners' English Corpus (CLEC). Zhen and Yang (2016) explored the main linguistic ideas of valency pattern and collovalency and their application value in English vocabulary teaching, highlighting the co-selection of lexis, structure and meaning. Then they introduced the methods to apply valency pattern and collovalency to teaching. Taking CONSIDER as an example, Zhen (2016) analyzed its valency patterns and translation equivalents in the English-Chinese parallel corpus to attempt to find the ideal translation unit. In addition, the valency pattern actually was restricted by its syntactic structure, which could distinguish its sense and influenced its translation equivalents. Based on this, he drew a conclusion that the words and their valency pattern constituted the ideal translation unit. And he (2017) also took the verb CONSIDER as an example and investigated how lexical meaning and its grammatical structures were from the perspective of valency pattern. Liu \& Du (2017) added the category of voice to the descriptive system and described valency patterns of the verb APPOINT appearing in the active sentences, passive sentences and others in BNC. Finally they analyzed the features of collocates and explored the relation among collocates, meaning and structure. Taking the verbs PROVIDE, HAPPEN, and LACK as examples, Zhang (2018) compared the features of these words' valency patterns and explored semantic and syntactic characteristics of node words, which were on the basis of Corpus of Contemporary American English and Ten-thousand English Compositions of Chinese Learners (TECCL).

\section{RESEARCH DESIGN}

\section{A. Research Questions}

Based on the purpose of the research, the paper aims to answer the following three questions:

(1) What valency sentence patterns does the English verb APPOINT have in active and passive sentences?

(2) What are the characteristics of these sentence valency patterns?

(3) What features do dependent constitutes of the English verb APPOINT indicate?

\section{B. Research Instruments}

In this study, AntConc 3.2.1 is employed as an auxiliary tool for analysis of collocation. AntConc is developed by Laurence Anthony of Waseda University in Japan. AntConc 3.2.1 was released on January 23, 2007, which is a revised version of the previous one. What's more, it is useful software in the research of corpus linguistics. It includes a powerful set of tools that are Concordance, Concordance Plot, File View, Clusters/N-Grams, Collocates, Word List and Keyword List. In the study, the function Concordance is primarily adopted.

\section{Research Procedures}

The first step is data collection. [appoint] is typed in the blank of interface of search of COCA and 300 samples can be extracted manually and randomly. Secondly, it is data analysis of samples. The author analyzes each concordance line and describe each valency sentence pattern of the verb APPOINT. However, some sentences, which are declarative sentences with interrogative, ellipsis, and attributive clauses, will be processed. For example, the clause "what John was appointed as" is rewritten into "John was appointed as what"; the attributive clause "Taskopruzade's father, who was appointed kadi of Aleppo by Selim I" is rewritten into "Taskopruzade's father was appointed kadi of Aleppo by Selim I". This helps to classify valency sentence patterns of the verb APPOINT. And the next step is to analyze the collocates of the verb APPOINT with the AntConc, by setting the span as 5L to 5R. And then characteristics of collocates will be summarized. Finally, the verbs ASSIGN and NOMINATE, near-synonyms of the verb APPOINT, are searched and 200 concordance lines of each verb are drawn. Valency sentence patterns of the two words are proposed and a comparison of identical sentence valency patterns of the three words is undertaken.

\section{RESULTS AND DisCUSSIONS}

The study retrieved the key word-APPOINT in the online corpus COCA, and selected 300 samples randomly, where the verb APPOINT is the predicate. And valency sentence patterns of the verb APPOINT in the active and 
passive sentences are described in the following part. And the feature of each complement of the verb APPOINT is analyzed. Finally, the overall feature of complements associated with the verb APPOINT is summarized.

\section{A. Valency Sentence Patterns of APPOINT in the Active Sentences}

Table II demonstrates seven valency sentence patterns identified for the verb APPOINT in the active sentences on the basis of the valency complement types mentioned in chapter three. In addition, the table contains respective frequency, percentage and example

TABLE II.

VALENCY SENTENCE PATTERNS Of APPOINT IN THE ACTIVE SENTENCES

\begin{tabular}{|l|c|c|c|c|}
\hline \multicolumn{1}{|c|}{ Valency sentence pattern } & Frequency & Proportion & \multicolumn{2}{c|}{ Divalent } \\
\hline Sub V Obj & 67 & $22.33 \%$ & $\begin{array}{l}\text { In 2001 President George W. Bush / appointed / James } \\
\text { Rogan. }\end{array}$ \\
\hline \multicolumn{3}{|c|}{ Trivalent } \\
\hline Sub V Obj vb-to-inf & 36 & $12 \%$ & $\begin{array}{l}\text { The state of Texas / appointed / an attorney / to } \\
\text { represent Ricky Eugene Kerr. }\end{array}$ \\
\hline Sub V Obj to N & 17 & $5.67 \%$ & $\begin{array}{l}\text { Republican President Dwight D. Eisenhower / } \\
\text { appointed / both Brennan and Warren / to the court. }\end{array}$ \\
\hline Sub V Obj nom-as & 16 & $5.33 \%$ & $\begin{array}{l}\text { Deputy Attorney General Rod Rosenstein / appointed / } \\
\text { Robert Mueller / as special counsel. }\end{array}$ \\
\hline Sub V Obj nom & 12 & $4 \%$ & $\begin{array}{l}\text { Rocky Mountain Internet Inc. / appointed / Melinda } \\
\text { Schild / vice president of human resources. }\end{array}$ \\
\hline Sub V Obj vb-to-be-nom & 5 & $1.67 \%$ & $\begin{array}{l}\text { Aristide / had appointed / Guy Malary / to be his } \\
\text { minister of justice. }\end{array}$ \\
\hline Tetravalent & \multicolumn{1}{|c|}{} \\
\hline Sub V Obj nom-as vb-to-inf & 2 & $0.67 \%$ & $\begin{array}{l}\text { Absent owners / could appoint / willing kinsmen / as } \\
\text { trustees / to oversee their lands and trees. }\end{array}$ \\
\hline
\end{tabular}

From the table II, we can directly see each valency sentence pattern of the verb APPOINT and respective frequency of occurrence and proportion in the three hundred concordance lines. As can be seen from the data in table II, the verb APPOINT in the active sentences has seven valency sentence patterns, including a divalent sentence pattern, five trivalent sentence patterns and one tetravalent sentence pattern. And the divalent pattern accounts for $22.33 \%$; the trivalent pattern is $28.67 \%$; the tetravalent pattern is $0.67 \%$. The data shows that the verb APPOINT in the active sentence is usually used in the trivalent and divalent pattern and rarely in the tetravalent pattern, which means that the verb APPOINT generally associates three or two complements in the actual use. Therefore, the tetravalent pattern of APPOINT will be excluded in the detailed analysis because of too low frequency of use. The data also suggests that in the active sentences, the highest frequency of occurrence is $\langle$ Sub V Obj $\rangle$, followed by $\langle$ Sub V Obj vb-to-inf $\rangle$; three trivalent valency sentence patterns, <Sub V Obj to N>, <Sub V Obj nom-as $>$ and <Sub V Obj nom>, virtually have the same frequency of use. What's more, the low frequency of $<$ Sub V Obj vb-to-be-nom $>$ and $<$ Sub V Obj as nom vb-to-inf $>$ implies uncommon usage. The following section will describe high-frequency valency sentence patterns.

(1) The Divalent Sentence Pattern in the Active Sentences

In the divalent structure the verb APPOINT associates a subject and an object complement, whose valency sentence pattern is described as Sub V Obj. Sub stands for subject complement and Obj is short for object complement. From the table above, it is found that the verb APPOINT solely has a divalent sentence pattern, which occurs most frequently and accounts for $43.22 \%$ in the active sentences. Therefore, English teachers should remind the students of paying more attention to the bivalent sentence pattern of the verb APPOINT in the second language vocabulary teaching. Except this, it is also found that there are significant features of dominant complements of the verb APPOINT in the bivalent sentence pattern.

Firstly, the feature of the subject complement is analyzed. After retrieving the note word APPOINT among the sixty seven concordance lines with AntConc, the result indicates that the subject complement primarily can be divided into five types, which are a personal pronoun, a position, a person's name, a position plus a person's name and others by frequency. By further calculations and analysis, the following data and rules are obtained. The personal pronoun occurs twenty one times, of which the highest-frequency pronoun he appears fourteen times. The second type is a noun meaning position, which is fifteen times. The position mainly constitutes state or government cadres, such as president, governor and mayor. And the word president occurs six times and governor and mayor are both three times in the divalent sentence pattern. And then the person's name appears eleven times. And the author finds that the person is almost prominent and possesses status and power, for instance, Donald Trump (the present president of the United States) and Guillaume Soro (the prime minister and president of the National Assembly of Cote d'Ivoire). The fourth type is the noun including a position plus a person's name, whose frequency of occurrence is low. It appears five times in total and shares the feature mentioned above. The last type is the noun that seems to have certain power. The word court and board occur five and three times respectively; the government sector also appears five times like State Legislate and Immigration Department. 
From the detailed analysis, the personal pronoun he is usually as the subject complement of the verb APPOINT in the divalent sentence pattern. And it can be seen that each type of the subject complement seems to have power except the first type the personal pronoun. Therefore, there is a hypothesis that the highest-frequency personal pronoun he should replace someone with status and power. To sum up, the subject complement in the divalent sentence pattern of the verb APPOINT is either a personal pronoun or a noun which possesses power.

Then it is found that the object complement has a significant characteristic. It is primarily a noun of profession or position. The profession occurs twenty times nearly accounting for one third. And it is made up of two kinds of occupation. One is judge appearing eleven times, the other is lawyer occurring four times. And the noun of position appears ten times. It is generally a government cadre, official or a company leader such as prime minster, auditor general, officer, manager. What's more, the person's name as the object complement occurs eight times. The person is usually prominent, like Dr. Shannon Hader who was the director of the HIV/AIDS administration at Washington's Department of Health, Zoe Baird who was an American lawyer and the president of the Markle Foundation. Another type of the object complement refers to a person or a group of members, like the member, committee, board, which has six occurrences. Finally, the personal pronoun can be the object complement in the valency sentence pattern $<$ Sub $\mathrm{V}$ Obj>. However, there is an exception that the time "the twenty second day" is also the object complement of the verb APPOINT, which is different from all kinds noted above. Then the meaning of the verb APPOINT in this sentence are likely not to be same as others. Generally the verb APPOINT refers to choosing someone for a position or a job, while its meaning is to arrange or decide a time for something to happen in the sentence "Johnson appointed the 22nd day". There is a hypothesis that it seems to determine the meaning of a polysemy through certain dominant complement. In summary, the profession and position are mainly as the object complement of the verb APPOINT in the valency sentence pattern <Sub V Obj>.

(2) The Trivalent Sentence Patterns in the Active Sentences

From the data of table II, it can be calculated that the trivalent sentence pattern occurs totally eighty six times and accounts for $55.48 \%$ in the active sentences. This reflects that the verb APPOINT often associates three complements when used in the active sentences. In addition, it can be clearly seen that the verb APPOINT has five trivalent sentence patterns, which are $\langle$ Sub V Obj vb-to-inf $>$, <Sub V Obj to N $>$, <Sub V Obj nom-as $\rangle$, <Sub V Obj nom $>$ and $\langle$ Sub V Obj vb-to-be-nom $>$ by frequency. The reference of Sub and Obj has been explained. Then the rest need to be expounded. $v b$-to-inf means a verbal complement with an infinitive; vb-to-be-nom stands for a verbal complement with to-be followed by a noun or a nominal phrase; to $N$ refers to a noun or noun phrase with the preposition to; nom-as refers to a nominal complement with the word as; nom is short for nominal complement. The complements in the trivalent sentence pattern of the verb APPOINT has a significant regularity as well. Each valency sentence pattern is analyzed in detail in the following section, but the last trivalent sentence pattern <Sub V Obj vb-to-be-nom> is excluded because of low frequency of occurrence.

$<$ Sub V Obj vb-to-inf > is the highest-frequency trivalent sentence pattern of the verb APPOINT in the active sentences. As can be seen, its realization includes a subject complement, an object complement and a verbal complement with an infinitive. The subject complement in the valency sentence pattern shares the features with that of the divalent sentence pattern. Similarly, it is primarily a personal pronoun, a position or profession, a person's name, a position plus a person's name, or a state apparatus. The top three are the personal pronoun, the person's name and the position plus a person's name. The person possesses power, which may be a state, government cadre or a company leader, like Sonny Perdue who served as the 81st governor of Georgia from 2003 to 2011.

The object complement is primarily a committee or an occupation. The committee occurs nine times and accounts for $45 \%$. And it is noted that the committee sometimes has a premodifier, for instance, mediating committee and faculty committee. Thus, the word committee or the phrase including the head committee is the object complement. The second kind of the object complement is a noun of profession appearing eight times. The profession is mainly the attorney. There are five occurrences of the person's name and four times of the post plus a person's name. The person is also important and well-known, like Paul Wolfwitz who was the president of the World Bank, United States Ambassador to Indonesia, US Deputy Secretary of Defence, and a dean of the Paul H. Nitze School of Advanced International Studies at Johns Hopkins University.

At last, the feature of the verb in the verbal complement is analyzed. It is found that the verb meaning that one is in charge of something accounts for one quarter in the valency sentence pattern. It can be the following words: head, serve as, chair, run. And the verb represent occurs three times. Except this, the verb can be the synonyms of the words investigate and monitor.

Seventeen occurrences of the trivalent sentence pattern $<$ Sub V Obj to $\mathrm{N}\rangle$ are found. It is realized by a subject complement, an object complement and a prepositional complement with the preposition to. The subject complement is primarily divided into three kinds by frequency: a personal pronoun, a post plus a person's name and a person's name. Its feature is very similar with the subject complement of last trivalent sentence pattern $<$ Sub V Obj vb-to-inf $>$. The object complement is mostly a person's name and a position. And both features are the same as what has been discussed above. The preposition to is followed by a noun or a noun phrase, which is court, board, or a government agency like the deliberative body and cabinet.

The trivalent sentence pattern <Sub V Obj nom-as > is realized by a subject complement, an object complement and a 
nominal complement with as. It is similar to the next trivalent sentence pattern <Sub V Obj nom>. Quirk et al. (1985) and Fischer (1997) states that nominal complement can occur either with as or without as, which suggests they are variations of the same valency sentence pattern. Therefore, the feature of complements in $<$ Sub V Obj nom $>$ is not analyzed in detail.

The question that arises is how to classify the word as. Does it belong to the part of speech preposition? Prepositions can be anaphorised with a paraphrase which is made up of the preposition plus a proper pronoun, as shown in example $\mathrm{k}$.

k. The general manager is thinking [of various influencing factors].
Anaphoristion
$\mathrm{He}$ is thinking [of it].

While, the nominal complement can be anaphorised with as such (Reichardt, 2014: 180). If the word as functions as a preposition, it is questionable for completion with a noun phrase, which is exemplified as follows.

m. Trump appointed a man as one of his advisers.
Anaphorisation :
as $\operatorname{him}(?)$ as it (?)
as such
-(?) Prepositional complement
- Nominal complement

In the example $\mathrm{m}$, if underlined part is identified through anaphorisation with as him or as it, it doesn't make sense. It suggests that as does not belong to preposition, but is a part of the nominal complement. Altman and Hahnemann (2010) make a claim that only particles which govern a case are classified as preposition in the analysis of German. The particle as represents a transposition to explain changes in the syntactic category of words in a sentence. Actually the nominal complement with as is also as predicative complement. Nevertheless, Reichardt (2014) proposes that it should categorise the structure under the sub-pattern $\langle\mathrm{Sub} \mathrm{V}$ Obj nom-as $\rangle$, since the structure solely occurs with verbs which govern a nominal complement.

The subject complement has a little difference with those in other valency sentence patterns mentioned above. Besides a person's name and a position plus a person's name, the subject complement contains a name of company as well. The object complement primarily includes a person's name and a personal pronoun. Seven occurrences of person's name accounts for $43.75 \%$. The personal pronoun occurs five times accounting for $33.33 \%$. The feature of the nominal complement is similar to that of the object complement in the divalent sentence pattern. It is made up of a noun of post or profession.

After analyzing the frequency and feature of each complement, this paper explores the characteristic of same type of dominant components of the verb APPOINT in the active sentences from the whole, which are the subject, object and nominal complement. To summarize, in the active sentences the subject complement of the verb APPOINT can be a personal pronoun, a person's name, a position, a position plus a person's name, a state or government organization, a name of company, the word court or a committee by possibility. Statistical results are presented below. The most frequent is the personal pronoun, which occurs thirty three times. And the personal pronoun he has nineteen occurrences and accounts for $57.58 \%$. The second highest-frequency is the person's name, whose occurrence is twenty nine. It has been discussed that the person is well-known and possesses power and status. The next is the noun of position as a subject complement, of which the word president occurs ten times and accounts for 47.62\%; the word mayor appears five times. Sixteen occurrences of a position plus a person's name are found. And the position is primarily made up of president (seven times) and governor (five times). In addition, a state or government organization can be a subject complement, which occurs sixteen times. Except those mentioned above, nine occurrences of a company's name, seven occurrences of the word court and six occurrences of a committee are found in the position of the subject complement of the verb APPOINT in the active sentences.

The object complement can be a position, a profession, a person's name, a personal pronoun, a member or a committee from the analysis of the whole. The highest-frequency of use is the position, which is used thirty one times. And then the occupation occurs twenty eight times, of which the judge accounts for $46.43 \%$ and the lawyer is $25 \%$. And twenty eight occurrences include person's names. The personal pronoun occurs eighteen times and the member and committee appear seventeen times. However, different valency sentence patterns have difference preference for the types. The first two valency sentence patterns, the divalent sentence pattern $\langle\mathrm{Sub} \mathrm{V} \mathrm{Obj}\rangle$ and the trivalent sentence pattern <Sub V Obj vb-to-inf $>$, have similar preference, whose object complement is generally a profession, a person's name or a committee. And it is easy to see that the last two trivalent sentence patterns have same types of the object complement. It is a person's name or a personal pronoun. Likewise, the feature of the nominal complement between two valency sentence patterns is common, which is a position or a profession. What's more, it is similar to that of the object complement in the divalent sentence pattern.

\section{B. Valency Sentence Patterns of APPOINT in the Passive Sentences}

There are a large number of valency sentence patterns of the verb APPOINT in the passive sentences. Table III shows specific valency sentence patterns identified for the verb APPOINT in the passive sentences. And the structure, frequency, proportion and example of each valency sentence pattern are presented as well. 
TABLE III.

VALENCy SENTENCE PATterns OF APPOINT In THE PASSIVE SENTENCES

\begin{tabular}{|c|c|c|c|}
\hline Valency sentence pattern & Frequency & Proportion & Example \\
\hline \multicolumn{4}{|c|}{ Monovalent } \\
\hline Sub be V-ed & 24 & $8 \%$ & David Olsen / was appointed. \\
\hline \multicolumn{4}{|c|}{ Divalent } \\
\hline Sub be V-ed nom & 36 & $12 \%$ & $\begin{array}{l}\text { Williams / is appointed / chief of the Los Angeles Police } \\
\text { Department. }\end{array}$ \\
\hline Sub be V-ed by N & 24 & $8 \%$ & Police chiefs / are appointed / by mayors. \\
\hline Sub be V-ed to $\mathrm{N}$ & 24 & $8 \%$ & $\begin{array}{l}\text { Dennis Monbarren / was appointed / to the new position of } \\
\text { Sunday editor. }\end{array}$ \\
\hline Sub be V-ed vb-to-inf & 12 & $4 \%$ & $\begin{array}{l}\text { Lee Brown / is appointed / to serve as the director of the } \\
\text { Office of National Drug Control Policy. }\end{array}$ \\
\hline Sub be V-ed nom-as & 4 & $1.33 \%$ & $\begin{array}{l}\text { The } 13 \text { Republican members of the House Judiciary } \\
\text { Committee / have been appointed / as managers of the } \\
\text { Senate's impeachment tria. }\end{array}$ \\
\hline Sub be V-ed vb-to-be-nom & 2 & $0.67 \%$ & I / was appointed / to be Mr. McVeigh's lawyer. \\
\hline \multicolumn{4}{|c|}{ Trivalent } \\
\hline Sub be V-ed to $\mathrm{N}$ by $\mathrm{N}$ & 5 & $1.67 \%$ & $\begin{array}{l}\text { Brandeis / was appointed / to the U.S. Supreme Court / by } \\
\text { President Woodrow Wilson in } 1916 .\end{array}$ \\
\hline Sub be V-ed by $\mathrm{N}$ to $\mathrm{N}$ & 2 & $0.67 \%$ & $\begin{array}{l}\text { Spahn / was appointed / by Mr. Obama / to a presidential } \\
\text { committee on the arts. }\end{array}$ \\
\hline Sub be V-ed by N vb-to-inf & 3 & $1 \%$ & I / was appointed / by the court / to represent a lady. \\
\hline Sub be V-ed nom to $\mathrm{N}$ & 3 & $1 \%$ & $\begin{array}{l}\text { Mike Masaoka of the JACL / was appointed / consultant / } \\
\text { to the President's Committee. }\end{array}$ \\
\hline Sub be V-ed nom by $\mathrm{N}$ & 2 & $0.67 \%$ & $\begin{array}{l}\text { She / had been appointed / an ambassador of culture by / } \\
\text { the Dominican Republic. }\end{array}$ \\
\hline $\begin{array}{l}\text { Sub be V-ed nom-as } \\
\text { vb-to-inf }\end{array}$ & 2 & $0.67 \%$ & $\begin{array}{l}\text { He / was appointed / as the first biologist / to head the } \\
\text { agency. }\end{array}$ \\
\hline Sub be V-ed nom-as to $\mathrm{N}$ & 1 & $0.33 \%$ & $\mathrm{He} /$ has been appointed / as the ambassador / to London. \\
\hline Sub be V-ed to N nom-as & 1 & $0.33 \%$ & $\begin{array}{l}\text { He / was appointed / to his third five-year term / as dean of } \\
\text { the Yale School of Architecture. }\end{array}$ \\
\hline
\end{tabular}

From the table III, it can be seen that there are plenty of valency sentence patterns of the verb APPOINT in the passive sentences. Even the number of the valency sentence pattern in the passive sentences is roughly as twice as that in the active sentences. However, five valency sentence patterns in the passive sentences are frequently employed,

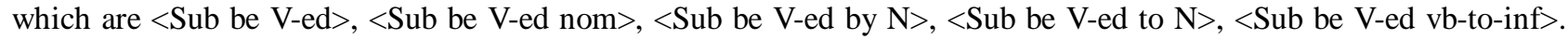
Especially all of the trivalent sentence patterns are rarely used. Eight trivalent sentence patterns were solely found in nineteen concordance lines in total, of which the most frequent one, 〈Sub be V-ed to $\mathrm{N}$ by $\mathrm{N}\rangle$, occurs five times. Therefore, these trivalent sentence patterns are presented in the table III but not analyzed in detailed further. The following section will analyze the five most frequent valency sentence patterns. And it is noted that four out of five most valency sentence patterns are divalent, which indicates that the verb APPOINT usually associates two complements in the passive sentences.

In addition, this following part also discusses the complements of the patterns of the verb APPOINT in the passive sentences. The complements of the verb APPOINT includes the subject complement, to N, by N,vb-to-inf, nom, nom-as. Each complement has been interpreted but by $N$, which means a noun or noun phrase after the preposition by often happening in the passive sentences. It is actually a part of grammatical structure. This paper aims to describe the valency patterns of the verb APPOINT in the sentences based on the corpus, so the frequency of occurrences should be an indicator. By $N$ associating the key word occurs thirty six times. In addition, the noun after the preposition by is equivalent of the subject complement in the active sentences, and they are the same semantically. Thus, it is described in the valency sentence patterns.

And from the specific trivalent sentence patterns presented in table III, it can be found that except the subject complement, the complement type of the verb APPOINT has a flexible position and can combine with other. The first trivalent sentence pattern $\langle$ Sub be $\mathrm{V}$-ed to $\mathrm{N}$ by $\mathrm{N}\rangle$ includes $t o N$ and by $N$. After their position being exchanged, a new trivalent sentence pattern $\langle$ Sub be $\mathrm{V}$-ed by $\mathrm{N}$ to $\mathrm{N}\rangle$ is generated. The combination of nom-as and to $N$ forms $<$ Sub be V-ed nom-as to $\mathrm{N}\rangle$ and $\langle$ Sub be V-ed to N nom-as $\rangle$. And $\langle$ Sub be V-ed nom-as vb-to-inf $\rangle$ is made up of a nominal complement with the word as and a verbal complement with an infinitive. The combinations of $v b$-to-inf and by $N$, by $N$ and nom, nom and to $N$ forms the trivalent sentence patterns 〈Sub be V-ed by N vb-to-inf〉, 〈Sub be V-ed nom by $\mathrm{N}\rangle$ and $<$ Sub be V-ed nom to $\mathrm{N}>$ respectively. This rule can be paid more attention in the actual use.

(1) The Monovalent Sentence Pattern in the Passive Sentences

The verb APPOINT solely has the monovalent sentence pattern <Sub be V-ed>. It occurs twenty four times and accounts for $16.55 \%$ of all valency sentence patterns in the passive sentences. It is used relatively frequently. The 
subject complement almost includes all kinds mentioned above. It can be a person's name, a profession, a member or committee, a position, a personal pronoun or a position plus a person's name. However, none of them is significantly high frequency. Their frequency is in the range of two to five. And there is an exception that "the dwellings" is also as the subject complement of the verb APPOINT, which is different from others. It has been mentioned two meanings of the verb APPOINT. It refers to choosing someone for a position or a job or arranging or deciding a time for something to happen. Obviously, the two meanings of the verb APPOINT are unreasonable in the sentence "The dwellings were well appointed.". The verb APPOINT has the similar meaning with the verb FURNISH there. It means that furniture and equipment are provided when you rent a room or house. This example further confirms the hypothesis that it seems to determine the meaning of a polysemy through certain dominant complement.

(2) The Divalent Sentence Patterns in the Passive Sentences

From the data of table III, there are six divalent sentence patterns of the verb APPOINT in the passive sentences, which occurs one hundred and two times and accounts for 70.34\%. This suggests that the verb APPOINT usually associates two complements when used in the passive sentences. It can be directly seen that the most frequent valency sentence pattern is the divalent sentence pattern $\langle$ Sub be $\mathrm{V}$-ed nom $>$. And the two divalent sentence patterns $<$ Sub be $\mathrm{V}$-ed by $\mathrm{N}>$ and $<$ Sub be $\mathrm{V}$-ed to $\mathrm{N}>$ are as frequent as the monovalent sentence pattern, which are the second place. The third place is <Sub be V-ed vb-to-inf>, whose frequency is half of the second. The rest two valency sentence patterns are excluded in the following analysis because of low frequency.

The highest-frequency valency sentence pattern <Sub be V-ed nom> is realized by a subject and a nominal complement. The subject complement primarily can be a person's name or a personal pronoun, of which both occur thirty three times and account for $91.67 \%$. Specifically, there are twenty one occurrence of the person's name and twelve occurrences of the personal pronoun. The nominal complement is largely a position, which occurs twenty eight times in thirty concordance lines. And the position is generally the leader of the government department, institute or company, like the chief of Police Department, the director of the Institute of Jewish Studies, the advertising development director. And the rank of certain occupation is as the nominal complement as well, such as bishop that is a clergyman of high rank in the Roman Catholic, Anglican, and Orthodox churches. Compared with the feature of the complements of the trivalent sentence pattern $<$ Sub V Obj nom $>$ in the active sentence, it is found that the feature of the two nominal complements is similar. In addition, there is similar characteristic between the subject complement of $<$ Sub be V-ed nom $>$ and the object complement of $\langle$ Sub V Obj nom $\rangle$. This rule should be predictable, because they belong to the same semantic role.

The realization of the divalent sentence pattern $\langle\mathrm{Sub}$ be $\mathrm{V}$-ed by $\mathrm{N}\rangle$ is a subject complement and a prepositional complement with the preposition by. Twenty four occurrences of the structure are found. The subject complement can be the following types: a member or committee, a position, a personal pronoun, a person's name or a profession. Their frequency ranges three to six. The noun after the preposition by is mainly a position, which occurs fourteen times and accounts for $58.33 \%$. And half of the positions are the president. In addition, there are four occurrences of a person's name.

The divalent sentence pattern of the verb APPOINT in the passive sentences corresponds to its structure $<$ Sub V Obj $>$ in the active sentences. Thus, the subject complement of <Sub be V-ed by N $>$ and the object complement of $<$ Sub $\mathrm{V} \mathrm{Obj}>$ share same semantic roles, so they have similar feature. The result shows that both of them include a profession, a position, a person's name or a member or committee. However, the frequency of occurrence of each type varies. And the noun of $<$ Sub be V-ed by $\mathrm{N}\rangle$ and the subject complement of $\langle\mathrm{Sub} \mathrm{V} \mathrm{Obj}\rangle$ share same semantic role, but have a difference. The noun after the preposition is solely a position or a person's name, whereas the subject complement of $<$ Sub V Obj> can be a personal pronoun, a position, a person's name, the word court or a government sector. The most frequent occurrence is the personal pronoun, followed by the position and the person's name. However, it has been discussed that each type of the subject complement of $\langle\mathrm{Sub} \mathrm{V} \mathrm{Obj}\rangle$ seems to have power except the personal pronoun. It can be inferred that the personal pronoun he should replace someone with status and power or certain position mentioned before the sentence. Therefore, the noun of $<$ Sub be V-ed by $\mathrm{N}>$ and the subject complement of $<$ Sub $\mathrm{V}$ Obj> share the feature.

The divalent sentence pattern <Sub be V-ed to $\mathrm{N}>$ occurs also twenty four times. Its realization is a subject complement and a prepositional complement with the preposition to. The subject complement is mostly a person's name, which occurs eleven times and approximately accounts for half. The profession and the personal pronoun can be as the subject complement. With regard to the noun after the preposition to, one thirds is the word position and post; a quarter is a committee.

$<$ Sub be V-ed vb-to-inf > is realized by a subject complement and a verbal complement with an infinitive. Its frequency of use is a little low. The structure is only found in twelve concordance lines. The subject complement is a person's name or a profession, which occurs five and four times respectively. And the profession is the lawyer. As for the verb in the verbal complement, it is generally the following verbs or their synonyms: represent, lead, serve as and investigate.

After discussing the frequency and feature of the relatively high-frequency valency sentence patterns of the verb APPOINT in the passive sentences, this section continues to explore the overall feature of the subject complement, the noun after the preposition by and nominal complement existing in the passive sentences. The rest complements, which 
are the noun after the preposition to and the verbal complement, will be analyzed combining with those occurring in the active sentences in the next section.

The subject complement is a person's name frequently. It occurs fifty three times and accounts for more than one third. The second type is a personal pronoun, which is observed as the subject complement of the verb APPOINT in thirty one concordance lines. The subject complement can be also the following three types occasionally: a profession, a position or a member or committee, whose frequency is fourteen, twelve and ten respectively. The prepositional complement by $N$ occurs thirty times in various valency sentence patterns of the verb APPOINT. And half of it is a position. Except this, it can be a person's name, a position plus a person's name or the word court. Finally, fifty one occurrences of the nominal complement appearing in the passive sentences are found. And there are thirty five concordance lines in which a position is as the nominal complement of the verb APPOINT.

\section{The Overall Feature of Complements of the Verb APPOINT}

From the data in table II and table III, it can be seen that the relatively most frequent valency sentence patterns of the verb APPOINT are Sub V Obj (22.33\%), Sub V Obj vb-to-inf (12\%), Sub be V-ed nom (12\%), Sub be V-ed (8\%), Sub be V-ed by $\mathrm{N}(8 \%)$ and Sub be V-ed to $\mathrm{N}(8 \%)$. And the verb APPOINT is almost evenly used in the active and passive sentences by calculating. There is no preference for active or passive voice according to the present data.

This section discusses the overall feature of common complements of the APPOINT occurring in the active and passive sentences, which are the subject, nominal, verbal complement and the prepositional complement with the preposition to. However, the subject complements cannot merge in active and passive sentences because of different semantic roles. The subject complement occurring in the active sentences includes a great number of types: a personal pronoun, a person's name, a position, a position plus a person's name, a state or government organization, a name of company, the word court or a committee. The subject complement existing in the passive sentences is primarily a person's name, which occurs fifty three times and accounts for over one third. The second type is a personal noun, which is found as the subject complement in thirty one concordance lines in the passive sentences. The subject complement can be a profession, a position, a member or committee as well. Actually, the subject complement in the passive sentences equals the object complement in the active sentences semantically. The feature of the object complement appearing in the active sentences has been analyzed above.

The nominal complement of the verb APPOINT is found in eighty four concordance lines. By analyzing and calculating, the result shows that it is largely a position, which occurs fifty one times and accounts for $60.71 \%$. And the position is generally the leader of the government department, institute or company. What's more, ten occurrences of profession are found as the nominal complement of the verb APPOINT, which is primarily the lawyer. This rule is not affected by the voice, which means that the feature of nominal complement is consistent in the active and passive voice.

In the prepositional complement the noun after the preposition to is the word post or position, or a board or committee relatively frequently. And it can be the word court, which occurs six times. Sometimes it can be a government department or a profession. Except those, the word bench occurs three times after the preposition to; the word term is found in two concordance lines. Occasionally, it can be someplace.

The overall feature of the verb in the verbal complement is the same as what it has been discussed above. The verbs, such as head, run and serve as, occur most frequently. They share the meaning that one is in charge of something. The verb represent appears frequently as well. In addition, the verb can be the synonyms of the words investigate and monitor.

\section{CONCLUSION}

\section{A. Research Findings}

This paper investigated valency sentence patterns of verbs from the perspective of syntactic valency including the category of voice, taking the verb English APPOINT as an example. And it analyzed dominated components of verbs with a corpus linguistic method of collocation. And it also explored if the valency sentence pattern is an indicator of substitution for near-synonym.

The results show that the verb APPOINT has twenty two identified valency sentence patterns totally in the active and passive sentences. The relatively most frequent valency sentence patterns of the verb APPOINT are $\langle$ Sub V Obj $>$ $(22.33 \%)$, <Sub V Obj vb-to-inf $>(12 \%),<$ Sub be V-ed nom $>(12 \%),<$ Sub be V-ed $>(8 \%),<$ Sub be V-ed by N $>(8 \%)$ and $<$ Sub be V-ed to $\mathrm{N}>(8 \%)$. Overall, the data do not suggest that the verb APPOINT has a preference for active or passive voice.

And there is regularity existing in each complement. The subject complement occurring in the active sentences can be a personal pronoun, a person's name, a position, a position plus a person's name, a state or government organization, a name of company, the word court or a committee. The object complement in the active sentences can be a position, a profession, a person's name, a personal pronoun, a member or a committee by frequency. The subject complement existing in the passive sentences is primarily a person's name, followed by a personal noun. Sometimes, the subject complement can be a profession, a position, a member or committee. The nominal complement of the verb APPOINT is largely a position, which is generally the leader of the government department, institute or company. It can be a profession as well, which is primarily the lawyer. The noun after the preposition by is generally a position, which 
accounts for a half. It can be a person's name, a position plus a person's name or the word court as well. The noun after the preposition to is the word post or position, or a board or committee relatively frequently. Except those, it can be a government department, a profession or the words court or bench. In the verbal complement, the verbs, such as head, run and serve as, occur most frequently. They share the meaning that one is in charge of something. The verb represent appears frequently as well. In addition, the verb can be the synonyms of the words investigate and monitor. It finds there is a relation among collocates, meaning and structure through the detailed analysis.

\section{B. Pedagogical Implications}

English belongs to the Indo-European language family, while Chinese belongs to the Sino-Tibetan language family. English learners, whose native language is Chinese, often misuse some words and grammatical structures due to differences of two languages. And English teachers explain words and structures by quoting some examples from dictionary and learners just memorize them by rote. Thus, it causes that there appears to be many mistakes in the actual use and learners lost interested in the English learning. This paper describes the valency sentence pattern of verbs and analyzes the features of the associated complements, which help students grasp the correct usage of the verb, reduce the use of inappropriate collocation and express clearly with complete sentences. Therefore, English teachers can focus on the sentences when teaching new words. Teachers can summarize the valency sentence patterns of certain word by collecting the authentic texts in the corpus and explains the features of associated complements. Learners will have a good command of complete expression of a word in a sentence and they gradually become interested in the language learning.

If English teacher summarizes the valency sentence patterns of every word, it will take a great amount of time and effort. Therefore, it is convenient to compile a dictionary about valency sentence patterns. And the valency sentence pattern constructs logical expressions, which can be as a framework and applied in the computational linguistics. If it is applied successfully, the valency sentence pattern of certain word will be retrieved and extracted from the corpus. And nowadays, there are a number of online corpora, which are updated every few years. This will be more convenient and provide latest texts.

\section{REFERENCES}

[1] Allerton Davide J. (1982). Valency and the English Verb. New York: Academic Press

[2] Chen Wenjie. (2009). A Brief Discussion about Corpus and Valency Grammar Studies. Journal of Guangxi University for Nationalities(Philosophy and Social Science Edition), (4): 145-148.

[3] Fischer K. (1997). German-English Verb Valency: Contrastive Analysis. Tübingen: Gunter Narr Verlag.

[4] Herbst T. (2009). Valency-Item-specificity and Idiom Principle. Exploring the Lexis Grammar Interface, (35): 49-68.

[5] Hunston, S. \& G. Francis. (2000). Pattern Grammar: A Corpus-driven Approach to the Lexical Grammar of English. Amsterdam: Benjamins.

[6] Liu Guobing. \& Du Yaping. (2017). A Corpus-driven Study of Verb Valency Pattern: The Case of the Verb APPOINT. Journal of Tianjin Foreign Studies University, (6): 1-7.

[7] Lu Jianming. (1996). A Study of Valence Grammar Theory and Teaching Chinese as a Foreign Language. Beijing: Beijing Language and Culture University.

[8] Quirk, R, Greenbaum, S, Leech, G, \& Svartvik, J. (1985) A Comprehensive Grammar of the English Language. London: Longman.

[9] Reichardt, R. (2014). Valency Sentence Patterns and Meaning Interpretation. Birmingham: University of Birmingham.

[10] Sinclair, J. (1991). Corpus, Concordance, Collocation. Oxford: Oxford University Press.

[11] Yuan Yulin. (2010). Study on the Valence of Chinese Verbs. Nannchang: Jiangxi Education Press.

[12] Zhang Lei. \& Wei Naixing. (2017). A Contrastive Study of the Local Grammar Patterns of Evaluation in Academic Research Articles across Western and Chinese Scholars. Journal of PLA University of Foreign Languages, (3): 10-18.

[13] Zhang Nannan. (2018). A Corpus-Driven Comparative Study of Verb Valency Patterns. Xinxiang: Henan Normal University.

[14] Zhen Fengchao. (2017). Exploring the Integration of Valency Pattern and Meaning: A Corpus-driven Approach. Foreign Language Research, (4): 13-18.

[15] Zhen Fengchao. (2016). A Corpus-Based Study of Translation Units from the Perspective of Valency Pattern. Foreign Language Teaching and Research, (3): 442-454.

[16] Zhen Fengchao. \& Yangfeng. (2016). Application of Valency Patterns and Collovalency to English Vocabulary Teaching: Ideas and Methods. Foreign Language World, (4): 35-42.

[17] Zhen Fengchao. \& Yangfeng. (2015). Verb Valency in Learner English: A Corpus-driven Study of CONSIDER. Journal of Foreign Languages, (6): 57-67.

[18] Zhi Youchang. \& Liu Wanyi. (2000). Valent Grammar and Valent Semantic Under specification. Journal of PLA University of Foreign Languages, (3): 10-18.

Guobing Liu was born in Henan, China. He received the master's degree in corpus linguistics in 2005. Now he is an EFL teacher at the Faculty of International Studies, Henan Normal University. He received the doctoral degree in corpus linguistics and computational linguistics in 2013. In recent years, he published several books and more than thirty academic papers in the key journals both home and abroad. His academic interests include corpus linguistics and foreign language teaching. 
Yaping Du was born in Henan, China. She will receive the master's degree in 2019. Now she studies at the Faculty of International Studies, Henan Normal University. She is interested in corpus linguistics and her master's thesis is also related to it. 